\title{
The pressure moments for two rigid spheres in low-Reynolds-number flow
}

\author{
D. J. Jeffrey \\ Department of Applied Mathematics, The University of Western Ontario, London, Ontario, N6A 5B9, \\ Canada \\ J. F. Morris and J. F. Brady \\ Department of Chemical Engineering, California Institute of Technology, Pasadena, California 91125
}

(Received 22 February 1993; accepted 2 June 1993)

The pressure moment of a rigid particle is defined to be the trace of the first moment of the surface stress acting on the particle. A Faxén law for the pressure moment of one spherical particle in a general low-Reynolds-number flow is found in terms of the ambient pressure, and the pressure moments of two rigid spheres immersed in a linear ambient flow are calculated using multipole expansions and lubrication theory. The results are expressed in terms of resistance functions, following the practice established in other interaction studics. The osmotic pressure in a dilute colloidal suspension at small Péclet number is then calculated, to second order in particle volume fraction, using these resistance functions. In a second application of the pressure moment, the suspension or particle-phase pressure, used in two-phase flow modeling, is calculated using Stokesian dynamics and results for the suspension pressure for a sheared cubic lattice are reported.

\section{INTRODUCTION}

A mechanical definition of the osmotic pressure in a colloidal dispersion has been given by Brady ${ }^{1}$ in terms of the hydrodynamic interactions among the suspended particles. In addition to the interactions already familiar in low-Reynolds-number hydrodynamics, a new "pressure interaction" must be defined. This new interaction is also needed in models of particulate two-phase systems, where particle-phase momentum balances necessitate the concept of a solid-phase pressure ${ }^{2-5}$ in order to complete the specification of the bulk stress. The "suspension pressure" thus introduced requires for its determination (by Stokesian dynamics ${ }^{6}$ simulations, for example) the hydrodynamic pressure interactions between particles. Examples of osmotic pressure and suspension pressure calculations are given at the end of this paper, after we have defined the pressure interaction precisely and established how it can be calculated.

In low-Reynolds-number hydrodynamics, interactions between particles are frequently specified by using the moments of the surface stress acting on each particle. The first moment of the stress has been decomposed in the past into an antisymmetric part, which equals the couple acting on the particle, and a traceless symmetric part called the stresslet. These two quantities have been tabulated for two rigid spheres in a series of papers summarized in Jeffrey and Onishi, ${ }^{7}$ Jeffrey, ${ }^{8}$ and Kim and Karrila. ${ }^{9}$ It is the trace of the first moment, however, that is needed for the pressure interactions, and this has not been studied before. We denote it by $S$ and define it for a specified particle as

$$
S=-\int \mathbf{x}^{\prime} \cdot \boldsymbol{\sigma} \cdot \mathbf{n} d A
$$

where $\mathbf{x}^{\prime}$ is the position vector measured relative to the particle center, and the integration is over the surface of the particle. The minus sign is included because the previous studies used it in their definitions; the integral can then be interpreted as the moment exerted by the sphere on the fluid. Such an interpretation and sign will be used in this paper in order to keep the equations similar to those already developed. Before we proceed with the calculations, it is important to pause for a moment and consider terminology. We should decide whether the term stresslet, which until now has referred to a traceless quantity, is to be expanded to include the trace of the first moment, or whether it should be left as the traceless quantity. After considering the equations that arise in the applications described later in this paper, we think that it is most convenient to make an analogy with the terminology used for the stress tensor. Thus the stress tensor has a nonzero trace, and when the traceless part of the stress tensor is referred to separately it is called the deviatoric stress. In the same way, a stresslet should have a nonzero trace, and if a traceless quantity is needed, it can be called either the traceless part of the stresslet, or the deviatoric stresslet.

\section{EXPRESSIONS FOR THE PRESSURE MOMENT OF A SPHERE}

First we derive a Faxén law for the pressure moment by using the reciprocal theorem. We start by recalling, 
that the pressure field $p(\mathbf{x})$ produced by a point force $\mathbf{F}$ acting at a point $\mathbf{y}$ is $p(\mathbf{x})=\mathbf{F} \cdot \mathbf{P}(\mathbf{x}-\mathbf{y})$, where

$$
\mathbf{P}(\mathbf{x})=\frac{1}{4 \pi} \frac{\mathbf{x}}{x^{3}} .
$$

Next we note that the velocity field $\mathbf{v}(\mathbf{x})$ around a point source of fluid of strength $Q$ located at the origin is

$$
\mathbf{v}=\frac{Q}{4 \pi} \frac{\mathbf{x}}{x^{3}}=Q \mathbf{P}(\mathbf{x}) \text {. }
$$

The reciprocal theorem is used in the form ${ }^{9}$

$$
\begin{aligned}
& \int \mathrm{v}_{1} \cdot\left(\sigma_{2} \cdot \mathbf{n}\right) d A+\int \mathbf{v}_{1} \cdot\left(\nabla \cdot \sigma_{2}\right) d V \\
& =\int \mathbf{v}_{2} \cdot\left(\sigma_{1} \cdot \mathbf{n}\right) d A+\int \mathbf{v}_{2} \cdot\left(\nabla \cdot \sigma_{1}\right) d V,
\end{aligned}
$$

where $\mathbf{n}$ is directed outward from the particle surface into the fluid. We take $\mathbf{v}_{1}$ to be the flow outside an expanding sphere whose radius is $a$ and whose rate of volume increase is $Q$; the sphere center is at the origin. For $\mathbf{v}_{2}$, we take the flow generatcd by a point force $\mathbf{F}$ at $\mathbf{y}$ when there is a sphere of constant size stationary at the origin. The reciprocal theorem becomes

$$
\begin{aligned}
& \int \frac{Q}{4 \pi} \frac{\mathbf{x}^{\prime}}{a^{3}} \cdot\left(\sigma_{2} \cdot \mathbf{n}\right) d A \\
& \quad+\int Q \mathbf{P}(\mathbf{x}) \cdot(-\mathbf{F}) \delta(\mathbf{x}-\mathbf{y}) d V(\mathbf{x})=0 .
\end{aligned}
$$

Simplifying further, we obtain

$$
\frac{Q}{4 \pi a^{3}}\left(-S_{2}\right)-Q \mathbf{F} \cdot \mathbf{P}(y)=0,
$$

and this gives an expression for the pressure moment as

$$
S_{2}=-4 \pi a^{3} \mathbf{F} \cdot \mathbf{P}(\mathbf{y}) \text {. }
$$

Now we observe that $-\mathbf{F} \cdot \mathbf{P}(\mathbf{y})=\mathbf{F} \cdot \mathbf{P}(-\mathbf{y})$ is the pressure that would exist at the origin if the point force were acting in the absence of the sphere. This is the "ambient" pressure as seen by the sphere, usually denoted $p^{\infty}(\mathbf{x}=0)$. Thus we obtain a Faxén law in the form

$$
S=4 \pi a^{3} p^{\infty}(\mathrm{x}=0) \text {. }
$$

The extension of this result to an arbitrary ambient flow follows by echoing Hinch's argument cited in Kim and Karrila ${ }^{9}$ that any ambient flow can be modeled by a suitable supcrposition of point forces.

Next, we obtain an exact expression for the pressure moment of one sphere in the presence of another in terms of multipole expansions. We follow the notation of Jeffrey and Onishi $^{7}$ throughout; equations taken from their paper will be labeled by JO. In terms of spherical coordinates $\left(\rho_{\alpha}, \theta_{\alpha}, \phi\right)$ centered on sphere $\alpha$, the quantity we wish to calculate is

$$
S=-a_{\alpha} \iint\left(-p+\mu \frac{\partial u}{\partial \rho_{\alpha}}\right) a_{\alpha}^{2} \sin \theta_{\alpha} d \theta_{\alpha} d \phi,
$$

where $u=\mathbf{u} \cdot \hat{\rho}_{\alpha}$. Using (JO 2.3) and (JO 2.1), we obtain

$$
a_{\alpha}^{3} \int p \sin \theta_{\alpha} d \theta_{\alpha} d \phi=4 \pi a_{\alpha}^{2} \mu \sum_{n=0}^{\infty} p_{0 n}^{(3-\alpha)} t_{\alpha} t_{3-\alpha}^{n} .
$$

A similar calculation based on [JO Eqs. (2.4), (2.7), and (2.1)] shows that the $\partial u / \partial \rho$ term in the integrand integrates to 0 . We should remember when comparing (2) with (1) that the coefficients $p_{m n}$ have the dimensions of velocity. Also, it is worth noting that the integration leads to a contribution $p_{00}$ from the sphere to its own pressure moment; however, this coefficient must always be zero, because it implies logarithmic velocities far from the sphere. The only contribution, then, is the pressure environment created by the second sphere. Since only $m=0$ terms appear in the expression, we can see that only axisymmetric motions will lead to nonzero pressure moments. This fact can also be deduced from general vector considerations.

\section{RESISTANCE FUNCTIONS}

As with the other interactions between spheres, the pressure moment can be expressed as a function of the velocities of the spheres and the ambient velocity field, given by

$$
\mathbf{U}(\mathbf{x})=\mathbf{U}_{\infty}+\boldsymbol{\Omega}_{\infty} \times_{\mathbf{x}}+\mathbf{E}_{\infty} \cdot \mathbf{x},
$$

with constant $\mathbf{U}_{\infty}, \boldsymbol{\Omega}_{\infty}$, and $\mathbf{E}_{\infty}$, in which case we are led to functions analogous to the resistance functions defined in earlier papers. We write

$$
\left(\begin{array}{l}
S_{1} \\
S_{2}
\end{array}\right)=\mu\left(\begin{array}{llll}
\mathbf{P}_{11} & \mathbf{P}_{12} & \mathbf{Q}_{11} & \mathbf{Q}_{12} \\
\mathbf{P}_{21} & \mathbf{P}_{22} & \mathbf{Q}_{21} & \mathbf{Q}_{22}
\end{array}\right) \cdot\left(\begin{array}{c}
\mathbf{U}_{1}-\mathbf{U}\left(\mathbf{x}_{1}\right) \\
\mathbf{U}_{2}-\mathbf{U}\left(\mathbf{x}_{2}\right) \\
\mathbf{E}_{1}-\mathbf{E}_{\infty} \\
\mathbf{E}_{2}-\mathbf{E}_{\infty}
\end{array}\right)
$$

The rotations of the spheres do not appear in the equation because it can be shown that they do not contribute to the trace. Since the only vector in the problem is $\mathbf{d}$, where

$$
\mathbf{d}=\left(\mathrm{x}_{2}-\mathrm{x}_{1}\right) /\left|\mathrm{x}_{2}-\mathrm{x}_{1}\right|
$$

is the dimensionless unit vector along the line of centers directed from particle 1 to particle 2 , clearly we have (including nondimensionalizing factors)

$$
\begin{aligned}
& \mathbf{P}_{\alpha \beta}=\pi\left(a_{\alpha}+a_{\beta}\right)^{2} X_{\alpha \beta}^{P} \mathbf{d}, \\
& \mathbf{Q}_{\alpha \beta}=\pi\left(a_{\alpha}+a_{\beta}\right)^{3} X_{\alpha \beta}^{Q}\left(\mathbf{d d}-\frac{1}{3} \mathbf{I}\right),
\end{aligned}
$$

and $X_{\alpha \beta}^{P}, X_{\alpha \beta}^{Q}$ are functions only of $s \equiv 2 r /\left(a_{\alpha}+a_{\beta}\right)$, where $r=\left|\mathbf{x}_{2}-\mathbf{x}_{1}\right|$. The other resistance functions that are contracted with the rate of strain have been made traceless, so it seems reasonable to follow this practice here. The only symmetries obeyed by the functions are labeling ones:

$$
\begin{aligned}
& X_{\alpha \beta}^{P}(s, \lambda)=-X_{(3-\alpha)(3-\beta)}^{P}\left(s, \lambda^{-1}\right), \\
& X_{\alpha \beta}^{Q}(s, \lambda)=X_{(3-\alpha)(3-\beta)}^{Q}\left(s, \lambda^{-1}\right) .
\end{aligned}
$$




\section{THE FUNCTIONS $X_{\alpha \beta}^{P}$}

\section{A. Method of reflections}

We start by deriving the first few terms by the method of reflections. Suppose sphere 1 is moving with velocity $\mathrm{U}_{1}=U \mathbf{d}$ toward sphere 2 . The pressure field at the center of sphere 2 is

$$
p=\frac{3}{2} a_{1} \mu U_{1} / r^{2}
$$

hence

$$
S_{2}=\pi\left(a_{1}+a_{2}\right)^{2} \mu X_{21}^{P} U_{1}=4 \pi a_{2}^{3}\left(\frac{3}{2}\right) a_{1} \mu U_{1} / r^{2},
$$

and therefore

$$
X_{21}^{P}=\frac{4}{(1+\lambda)^{2}} \frac{6 \lambda^{3}}{(1+\lambda)^{2} s^{2}} .
$$

And, from the above relations,

$$
X_{12}^{P}=-\frac{4}{(1+\lambda)^{2}} \frac{6 \lambda}{(1+\lambda)^{2} s^{2}} .
$$

Sphere 2 responds to the ambient velocity induced near it by sphere 1 by exerting a force on the fluid; hence

$$
S_{1}=4 \pi a_{1}^{2} \mu X{ }_{11}^{P} U_{1}=4 \pi a_{1}^{3}\left(\frac{3}{2}\right)\left(a_{2} / r^{2}\right) \mu\left(3 a_{1} / 2 r\right) U_{1}
$$

and

$$
X_{11}^{P}=\frac{18 \lambda}{(1+\lambda)^{3} s^{3}}
$$

\section{B. Twin multipole expansions}

These functions can be calculated using the results obtained in JO Sec. III. In terms of $P_{n p q}$ defined in [JO, Eqs. (3.4)-(3.9)], we have

$$
X_{11}^{P}-\frac{1}{4}(1+\lambda)^{2} X_{12}^{P}=\sum_{n=1}^{\infty} \sum_{p=0}^{\infty} \sum_{q=0}^{\infty} \frac{3}{2} P_{n p q} t_{2}^{p+n_{1}^{q}}{ }_{1}^{q+1}
$$

For the complementary problem defined in [JO, Eq. (3.11)], we have

$$
\begin{aligned}
X_{11}^{P} & +\frac{1}{4}(1+\lambda)^{2} X_{12}^{P} \\
& =\sum_{n=1}^{\infty} \sum_{p=0}^{\infty} \sum_{q=0}^{\infty}(-1)^{n+p+q+2} \frac{3}{2} P_{n p q} t_{2}^{p+n} t_{1}+1 .
\end{aligned}
$$

From these equations, we see that the pattern observed with the earlier functions continues to hold, namely that the even and odd powers of $s$ divide between the functions. Thus

$$
\begin{aligned}
X_{11}^{P}(s, \lambda)= & \sum_{\substack{m=1 \\
m \text { odd }}}^{\infty} \sum_{q=1}^{m-1} \sum_{n=1}^{(q+1) / 2} \frac{3}{2} P_{n(q-n)(m-q-1)} \lambda^{q} \\
& \times \frac{2^{m}}{(1+\lambda)^{m} s^{m}}=\sum_{\substack{m=1 \\
m \text { odd }}}^{\infty} \frac{f_{m}(\lambda)}{(1+\lambda)^{m} s^{m}}
\end{aligned}
$$

TABLE I. Values of the function $P_{\alpha \beta}^{X}(\lambda)$, with $\lambda$ the size ratio of the two spheres, appearing in the asymptotic form of $X_{\alpha \beta}^{P}$ for small separation.

\begin{tabular}{rrrrr}
\hline \multicolumn{1}{r}{$\lambda$} & \multicolumn{1}{c}{$P_{11}^{X}$} & \multicolumn{1}{c}{$P_{12}^{X}$} & $P_{21}^{X}$ & \multicolumn{1}{c}{$P_{22}^{X}$} \\
\hline 1 & -0.0118 & -0.1435 & 0.1435 & 0.0118 \\
2 & 0.0930 & -0.1279 & 0.2581 & -0.0634 \\
3 & 0.3236 & -0.1283 & 0.3199 & -0.0963 \\
4 & 0.5662 & -0.1186 & 0.3337 & -0.1024 \\
5 & 0.7925 & -0.1059 & 0.3249 & -0.0988 \\
10 & 1.6347 & -0.0576 & 0.2286 & -0.0653 \\
20 & 2.5543 & -0.0237 & 0.1193 & -0.0322 \\
100 & 4.0499 & -0.0016 & 0.0114 & -0.0029 \\
\hline \hline
\end{tabular}

$$
X_{12}^{P}(s, \lambda)=\frac{-4}{(1+\lambda)^{2}} \sum_{\substack{m=2 \\ m \text { even }}}^{\infty} \frac{f_{m}(\lambda)}{(1+\lambda)^{m} s^{m}}
$$

where

$$
\begin{aligned}
& f_{0}=f_{1}=0, \quad f_{2}=6 \lambda, \quad f_{3}=18 \lambda, \\
& f_{4}=54 \lambda^{2}, \quad f_{5}=-24 \lambda+162 \lambda^{2}+216 \lambda^{3}, \\
& f_{6}=216 \lambda^{2}+486 \lambda^{3}+576 \lambda^{4}, \\
& f_{7}=432 \lambda^{2}+498 \lambda^{3}+2592 \lambda^{4}+1440 \lambda^{5}, \\
& f_{8}=864 \lambda^{2}+3888 \lambda^{3}+7446 \lambda^{4}+7128 \lambda^{5}+3456 \lambda^{6} .
\end{aligned}
$$

\section{Lubrication theory}

The flow between nearly touching spheres has been studied in Jeffrey and Corless ${ }^{11}$ and Jeffrey. ${ }^{12}$ Solutions based on an expansion in the small parameter $\epsilon$ were given there. In the latter paper, it was shown that, when higher orders are included, not all quantities can be approximated successfully by considering only the flow in the gap. We follow the method given there to circumvent that difficulty by writing the pressure moment as

$$
\begin{aligned}
S_{1} & =-\int \mathbf{x}^{\prime} \cdot \boldsymbol{\sigma} \cdot \mathbf{n} d A \\
& =a_{1} \mathbf{F} \cdot \mathbf{d}-a_{1} \int(\mathbf{n}+\mathbf{d}) \cdot \boldsymbol{\sigma} \cdot \mathbf{n} d A .
\end{aligned}
$$

Using the known result for $\mathbf{F}$ and integrating the previously obtained solution (which had been found using the algebra system Maple, and which was therefore easy to reprogram), we obtain

$$
X_{11}^{P}=g_{1} \xi^{-1}+g_{2} \ln \xi^{-1}+P_{11}^{X}(\lambda)+g_{3} \xi \ln \xi^{-1}+O(\xi)
$$

and

$$
\begin{aligned}
\frac{1}{4}(1+\lambda)^{2} X_{12}^{P}= & -g_{1} \xi^{-1}-g_{2} \ln \xi^{-1}+\frac{1}{4}(1+\lambda)^{2} P_{12}^{X}(\lambda) \\
& -g_{3} \xi \ln \xi^{-1}+O(\xi),
\end{aligned}
$$

where 


$$
\begin{aligned}
& g_{1}=3 \lambda^{2} /(1+\lambda)^{3}, \quad g_{2}=\frac{3}{10}\left(\lambda-4 \lambda^{2}\right) /(1+\lambda)^{2}, \\
& g_{3}=\frac{5-97 \lambda+64 \lambda^{2}-44 \lambda^{3}+\lambda^{4}}{140(1+\lambda)^{2}},
\end{aligned}
$$

the nondimensional gap width is $\xi=s-2$ and the $P_{\alpha \beta}^{\dot{X}}$ are functions that we shall tabulate here (cf. Table I). We can notice that, as with the $X^{A}$ functions, the singular terms cancel if the two spheres have the same velocity.

\section{Arbitrary separations}

The singularities cause slow convergence of the series (6a) and (6b) when $s$ is near 2 . We remove them from the series by giving the $g_{i}$ appearing in $(7 a)-(7 b)$ the values defined by (8) and then adding the left-hand side of (7a) to (6a), while at the same time subtracting the right-hand side of (7a) from (6a). If $\widetilde{f}(\lambda)=2^{-m} f(\lambda)$, we write

$$
\begin{aligned}
X_{11}^{P}= & g_{1} \frac{2 s}{s^{2}-4}+\left[g_{2}+g_{3}\left(\frac{1}{4} s^{2}-1\right)\right] \ln \frac{s+2}{s-2}-g_{3} s \\
& +\sum_{\substack{m=1 \\
m \text { odd }}}^{\infty}\left(\frac{\widetilde{f}_{m}(\lambda)}{(1+\lambda)^{m}}-g_{1}-\frac{2 g_{2}}{m}+\frac{4 g_{3}}{m(m+2)}\right)\left(\frac{2}{s}\right)^{m} .
\end{aligned}
$$

Mathematically this is equivalent to (6a), but numerically the rate of convergence has improved, because the coeffcients of $s^{-m}$ now decay faster by a factor $m^{-2}$, owing to cancellation. Similarly,

$$
\begin{aligned}
& \frac{(1+\lambda)^{2}}{4} X_{12}^{P} \\
& =-g_{1} \frac{4}{s^{2}-4}-\left[g_{2}+g_{3}\left(\frac{1}{4} s^{2}-1\right)\right] \ln \frac{s^{2}}{s^{2}-4}+g_{3} \\
& \quad-\sum_{\substack{m=2 \\
m \text { even }}}^{\infty}\left(\frac{\tilde{f}_{m}(\lambda)}{(1+\lambda)^{m}}-g_{1}-\frac{2 g_{2}}{m}+\frac{4 g_{3}}{m(m+2)}\right)\left(\frac{2}{s}\right)^{m} .
\end{aligned}
$$

Numerical tabulations of $X_{\alpha \beta}^{P}$ are not given because the expressions and data given above are accurate to at least two significant digits for all $s$. We do tabulate the $P_{\alpha \beta}^{X}(\lambda)$, however, because they provide a good test of the convergence of the series, as well as being useful in studies of nearly touching spheres. Expanding the leading terms in (9a) and comparing with (7a), we obtain

$$
\begin{aligned}
P_{11}^{X}= & \frac{1}{4} g_{1}+g_{2} \ln 4-2 g_{3} \\
& +\sum_{\substack{m=1 \\
m \text { odd }}}^{\infty}\left(\frac{\widetilde{f}_{m}(\lambda)}{(1+\lambda)^{m}}-g_{1}-\frac{2 g_{2}}{m}+\frac{4 g_{3}}{m(m+2)}\right),
\end{aligned}
$$

and similarly

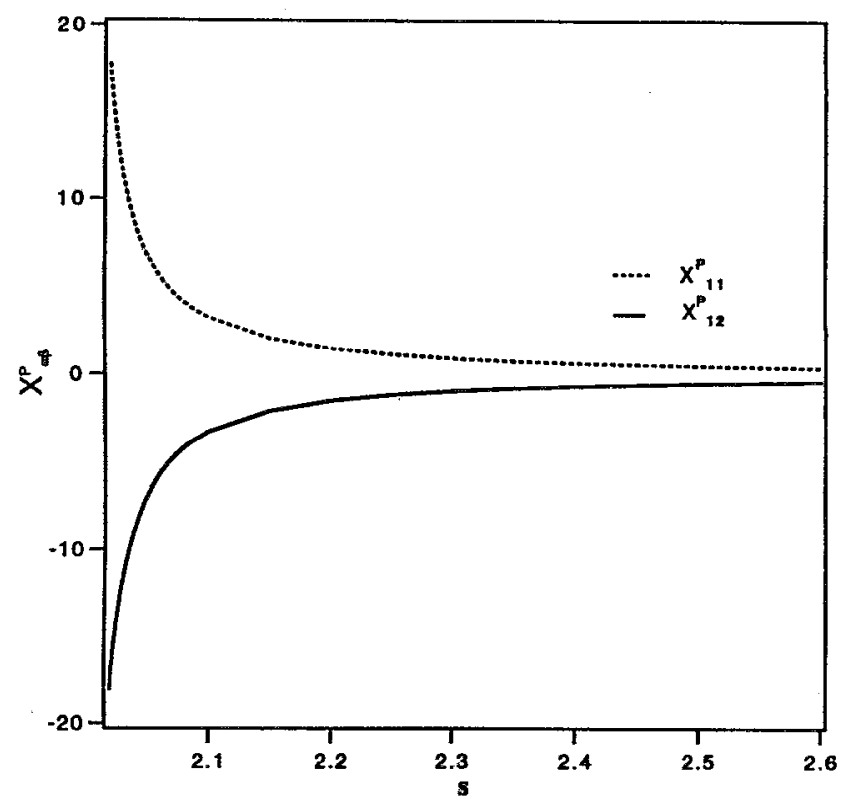

FIG. 1. The functions $X_{11}^{P}$ and $X_{12}^{P}$ relating velocities to the trace of the first moment of the surface force distribution for equal-sized spheres are plotted against the separation distance scaled to the particle radius.

$$
\begin{aligned}
\frac{(1+\lambda)^{2}}{4} P_{12}^{X}= & \frac{1}{4} g_{1}+g_{3}-\sum_{\substack{m=2 \\
m \text { even }}}^{\infty}\left(\frac{\widetilde{f}_{m}(\lambda)}{(1+\lambda)^{m}}-g_{1}-\frac{2 g_{2}}{m}\right. \\
& \left.+\frac{4 g_{3}}{m(m+2)}\right)
\end{aligned}
$$

\section{E. Results for $\boldsymbol{X}_{\alpha \beta}^{P}$}

To illustrate the behavior of the pressure moment, in Fig. 1 we plot $X_{11}^{P}$ and $X_{12}^{P}$ as a function of $s$ for the case of identical spheres $\lambda=1$. Note that the singular behavior of $X_{11}^{P}$ (and $X_{12}^{P}$ ) is proportional to the corresponding resistance functions $X_{11}^{A}$, etc., relating forces to translational velocities. In Table I we give the results of summing the series (10a)-(10b) for $P_{\alpha \beta}^{X}$ to 300 terms. We can estimatė the rate of convergence by making a comparison with sums to 200 terms, and that shows that the results are generally accurate to four significant figures.

\section{THE FUNCTIONS $x_{\alpha \beta}^{0}$}

\section{A. Method of reflections}

If sphere 1 deforms at a rate $\mathbf{E}_{1}=E_{1}\left(\mathbf{k} \mathbf{k}-\frac{1}{3} \mathbf{I}\right)$, the pressure at the center of sphere 2 is

$$
p=\frac{10}{3} a_{1}^{3} \mu E_{1} / r^{3} .
$$

Hence,

$$
S_{2}=4 \pi a_{2}^{3} \frac{10}{3} a_{1}^{3} \mu E_{1} / r^{3}=\pi\left(a_{1}+a_{2}\right)^{3} \mu X \frac{Q_{2} \frac{2}{3} E_{1},}{}
$$

and therefore

$$
X_{21}^{Q}=\frac{8}{(1+\lambda)^{3}} \frac{20 \lambda^{3}}{(1+\lambda)^{3} s^{3}} .
$$


There will be an ambient flow $\left(\frac{5}{3}\right) a_{1} E_{1}\left(a_{1}^{2} / r^{2}\right) \mathbf{d}$ created by sphere 1, which will lead to an induced $S_{1}$ of

$$
S_{1}=8 \pi a_{1}^{3} \mu X_{11}^{Q}\left(\frac{2}{3}\right) E_{1}=-\pi\left(a_{1}+a_{2}\right)^{2} X_{12}^{P}\left(\frac{5}{3}\right)\left(a_{1}^{3} / r^{2}\right) E_{1},
$$

implying

$$
X_{11}^{Q}=\frac{30 \lambda}{(1+\lambda)^{4} s^{4}} .
$$

\section{B. Twin multipole expansions}

The calculation in Jeffrey ${ }^{8}$ used the problem

$$
\mathbf{E}_{\alpha}=E_{\alpha}\left(\mathbf{k k}-\frac{1}{3} \mathbf{I}\right),
$$

together with the condition

$$
a_{1} E_{1}=a_{2} E_{2} \text {. }
$$

This means that

$$
S_{1}=8 \pi \mu a_{1}^{3} X \underset{113}{Q \frac{2}{3}} E_{1}+\pi \mu\left(a_{1}+a_{2}\right)^{3} X_{12 \frac{2}{3}}^{Q 2} E_{2},
$$

and therefore

$$
S_{1}=\frac{16}{3} \pi \mu a_{1}^{3} E_{1}\left(X_{11}^{Q}+\frac{(1+\lambda)^{3}}{8 \lambda} X_{12}^{Q}\right)
$$

From this we find

$$
X_{11}^{Q}+\frac{(1+\lambda)^{3}}{8 \lambda} X_{12}^{Q}=\sum_{n=1}^{\infty} \sum_{p=0}^{\infty} \sum_{q=0}^{\infty} \frac{5}{2} P_{n p q} t_{2}^{p+n} t_{1}^{q+1} .
$$

The complementary problem adds a factor $(-1)^{n+p+q+3}$ to the summation, so we conclude

$$
X_{11}^{Q}(s, \lambda)=\sum_{\substack{m=0 \\ m \text { even }}}^{\infty} \frac{f_{m}(\lambda)}{(1+\lambda)^{m} s^{m}}
$$

and

$$
X \frac{Q}{12}(s, \lambda)=\frac{8}{(1+\lambda)^{3}} \sum_{\substack{m=1 \\ m \text { odd }}}^{\infty} \frac{f_{m}(\lambda)}{(1+\lambda)^{m} s^{m}},
$$

where

$$
\begin{aligned}
& f_{0}=f_{1}=f_{2}=0, \quad f_{3}=20 \lambda^{3}, \quad f_{4}=30 \lambda \\
& f_{5}=90 \lambda^{4}, \quad f_{6}=-72 \lambda+270 \lambda^{2}+680 \lambda^{3}, \\
& f_{7}=720 \lambda^{4}+810 \lambda^{5}+864 \lambda^{6}, \\
& f_{8}=864 \lambda^{2}+3888 \lambda^{3}+7446 \lambda^{4}+7128 \lambda^{5}+3456 \lambda^{6} .
\end{aligned}
$$

\section{Lubrication theory}

We expect

$$
\begin{aligned}
8 \pi a_{1}^{3} \mu X_{11}^{Q \frac{2}{3}} E_{1} & =a_{1} \mathbf{F} \cdot \mathbf{d}-a_{1} \int(\mathbf{n}+\mathbf{d}) \cdot \boldsymbol{\sigma} \cdot \mathbf{n} d A, \\
& =4 \pi a_{1}^{3} X_{11}^{G} E_{z z}-a_{1} \int(\mathbf{n}+\mathbf{d}) \cdot \boldsymbol{\sigma} \cdot \mathbf{n} d A,
\end{aligned}
$$

and we obtain

$$
X \underset{11}{Q}=g_{1} \xi^{-1}+g_{2} \ln \xi^{-1}+Q_{11}^{X}+g_{3} \xi \ln \xi^{-1},
$$

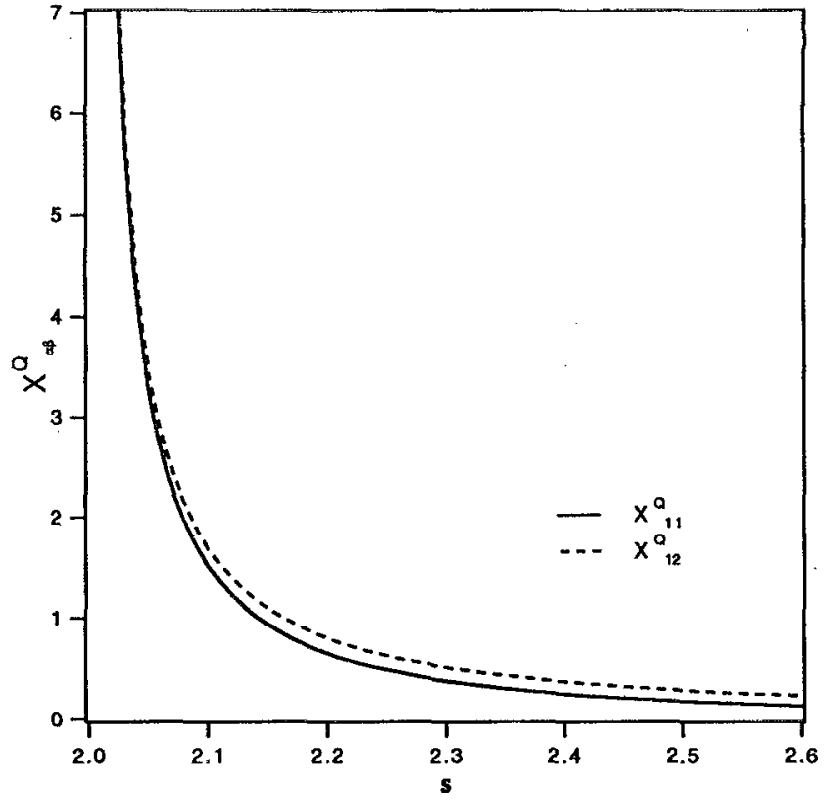

FIG. 2. The functions $X \rho_{1}$ and $X$ \& relating the rate of strain to the trace of the first moment of the surface force distribution for equal-sized spheres are plotted against the separation distance scaled to the particle radius.

$$
X_{12}^{Q}=g_{4} \xi^{-1}+g_{5} \ln \xi^{-1}+Q_{12}^{X}+g_{6} \xi \ln \xi^{-1},
$$

where

$g_{1}=\frac{3}{2} \lambda^{2} /(1+\lambda)^{3}, \quad g_{2}=\frac{3}{20}\left(\lambda+2 \lambda^{2}-9 \lambda^{3}\right) /(1+\lambda)^{3}$, $g_{3}=\frac{5-\lambda-201 \lambda^{2}+251 \lambda^{3}-184 \lambda^{4}}{280(1+\lambda)^{3}}, \quad g_{4}=12 \lambda^{3} /(1+\lambda)^{6}$,

$g_{5}=\frac{12}{5}\left(-2 \lambda^{2}+\lambda^{3}-2 \lambda^{4}\right) /(1+\lambda)^{6}$,

$g_{6}=\frac{-65 \lambda+34 \lambda^{2}-411 \lambda^{3}+76 \lambda^{4}-44 \lambda^{5}}{35(1+\lambda)^{6}}$.

As a check on our working we have the identity $4 X_{11}^{P}(1+\lambda)\left(1+\frac{1}{2} \xi\right)-8 X_{11}^{Q}-(1+\lambda)^{3} X_{12}^{Q}=O(1)$.

\section{Arbitrary separations}

As before, we have the following expressions for $Q X$ and $Q_{12}^{X}$ defined in Eqs. (12a)-(12b):

$$
\begin{aligned}
Q_{11}^{X}= & -\frac{1}{4} g_{1}-g_{3} \\
& +\sum_{\substack{m=2 \\
m \text { even }}}^{\infty}\left(\frac{\widetilde{f}_{m}(\lambda)}{(1+\lambda)^{m}}-g_{1}-\frac{2 g_{2}}{m}+\frac{4 g_{3}}{m(m+2)}\right)
\end{aligned}
$$

and

$$
\begin{aligned}
\frac{(1+\lambda)^{3}}{8} Q_{12}^{X}= & \frac{1}{4} g_{1}+g_{2} \ln 4-2 g_{3} \\
& +\sum_{\substack{m=1 \\
m \text { odd }}}^{\infty}\left(\frac{\widetilde{f}_{m}(\lambda)}{(1+\lambda)^{m}}-g_{1}-\frac{2 g_{2}}{m}+\frac{4 g_{3}}{m(m+2)}\right)
\end{aligned}
$$


TABLE II. Values of the function $Q_{\alpha \beta}^{X}(\lambda)$, with $\lambda$ the size ratio of the two spheres, appearing in the asymptotic form of $X_{\alpha \beta}^{Q}$ for small separation.

\begin{tabular}{rrrcr}
\hline \hline \multicolumn{1}{c}{$\lambda$} & $Q_{11}^{X}$ & $Q_{12}^{X}$ & $Q_{21}^{X}$ & \multicolumn{1}{c}{$Q_{22}^{X}$} \\
\hline 1 & -0.0799 & 0.1329 & 0.1329 & -0.0799 \\
2 & 0.1581 & 0.1314 & 0.1332 & -0.0413 \\
3 & 0.5166 & 0.1219 & 0.1263 & -0.0000 \\
4 & 0.8686 & 0.1088 & 0.1146 & 0.0186 \\
5 & 1.1875 & 0.0959 & 0.1020 & 0.0262 \\
10 & 2.3350 & 0.0528 & 0.0571 & 0.0254 \\
20 & 3.5543 & 0.0226 & 0.0244 & 0.0138 \\
100 & 6.2409 & 0.0018 & 0.0020 & 0.0013 \\
\hline \hline
\end{tabular}

\section{E. Results for $X_{\alpha \beta}^{a}$}

To illustrate the behavior of the pressure moment, in Fig. 2 we plot $X{ }_{11}^{Q}$ and $X \underset{12}{Q}$ as a function of $s$ for the case of identical spheres $\lambda=1$. Again, the singular behavior as $s \rightarrow 2$ is proportional to the corresponding force to rate of strain coupling $X_{11}^{G}$, etc. In Table II we give the results of summing the series (13a)-(13b) for $Q_{\alpha \beta}^{X}$ to 300 terms. We can estimate the rate of convergence by making a comparison with sums to 200 terms, and that shows that the results are generally accurate to four significant figures.

\section{OSMOTIC PRESSURE IN A DILUTE SUSPENSION}

We determine the correction to the osmotic pressure of a dilute suspension of Brownian hard spheres. Batchelor ${ }^{13}$ determined the Brownian contribution to the bulk deviatoric stress, and here we complete that calculation for the isotropic stress.

In the presence of Brownian motion there are two contributions to the bulk stress, which can be written as ${ }^{1,6}$

$$
\langle\Sigma\rangle=-n k T \mathbf{I}+2 \mu\langle\mathbf{E}\rangle+n\left[\left\langle\mathbf{S}^{E}\right\rangle+\left\langle\mathbf{S}^{B}\right\rangle\right],
$$

where $\langle\mathbf{E}\rangle$ is the bulk rate of strain in the material and the rate-of-strain and Brownian stresslets are given by

$$
\begin{aligned}
& \left\langle\mathbf{S}^{E}\right\rangle=-\left\langle\mathbf{R}_{S U} \cdot \mathbf{R}_{F U}^{-1} \cdot \mathbf{R}_{F E}-\mathbf{R}_{S E}\right\rangle:\langle\mathbf{E}\rangle, \\
& \left\langle\mathbf{S}^{B}\right\rangle=-k T\left\langle\nabla \cdot\left(\mathbf{R}_{S U} \cdot \mathbf{R}_{F U}^{-1}\right)\right\rangle .
\end{aligned}
$$

In (15)-(16), $\mathbf{R}_{F U}, \mathbf{R}_{S U}$, etc. are the hydrodynamic resistance tensors that couple the hydrodynamic force/torque to the particle velocities $\left(\mathbf{R}_{F U}\right)$, the stresslets to the velocities $\left(\mathbf{R}_{S U}\right)$, etc. (see Brady and Bossis ${ }^{6}$ ). Here we have assumed that the stresslets are not traceless, in contrast to the convention in the past, because we wish to determine the trace or pressure. In (14)-(16), $k$ is Boltzmann's constant, $T$ is the absolute temperature, and $n$ is the number density of suspended particles.

The osmotic or suspension pressure, $\Pi$, is defined, mechanically, as minus one-third the trace of the bulk stress:

$$
\Pi=n k T+n k T\langle\nabla \cdot \mathbf{A}\rangle+n\langle\mathrm{~B}\rangle:\langle\mathbf{E}\rangle,
$$

where the hydrodynamic functions $\mathbf{A}$ and $\mathbf{B}$ are defined by

$$
\begin{aligned}
& \mathbf{A}=\frac{1}{3} \mathbf{P} \cdot \mathbf{R}_{F U}^{-1}, \\
& \mathbf{B}=\frac{1}{3}\left(\mathbf{P} \cdot \mathbf{R}_{F U}^{-1} \cdot \mathbf{R}_{F E}-\mathbf{Q}\right),
\end{aligned}
$$

and $\mathbf{P}$ and $\mathbf{Q}$ are the pressure functions defined in (3). The procedure of obtaining (19) from (15) shows that $\mathbf{P}=\mathbf{I}: \mathbf{R}_{S U}$ and $\mathbf{Q}=\mathbf{I}: \mathbf{R}_{S E}$, where the resistance functions $\mathbf{R}_{S U}$ and $\mathbf{R}_{S E}$ are regarded as the complete relations between particle kinematics and the stresslets, as we are not restricting the hydrodynamic stress to be traceless.

Under equilibrium conditions (when $\langle\mathbf{E}\rangle \equiv 0$ ), the osmotic pressure is given by the first two terms on the righthand side of (17), which may be shown to give precisely the osmotic pressure defined thermodynamically in terms of the free energy or interparticle potential. ${ }^{1}$ When a shear flow is applied, the suspension microstructure is distorted from its equilibrium isotropic form, and both the Brownian, $n k T\langle\nabla \cdot \mathbf{A}\rangle$, and rate-of-strain, $n\langle\mathbf{B}\rangle:\langle\mathbf{E}\rangle$, contributions to the osmotic pressure, are nonzero. When the amplitude of the shearing motion is small, the distortion of the structure is linear and proportional to $\langle\mathbf{E}\rangle$, which occurs to leading order in the Péclet number, $\mathrm{Pe}=6 \pi \mu a^{3} \dot{\gamma} / k T$, where $j=|\langle\mathbf{E}\rangle|$, some measure of the rate of strain, for example, the largest principal strain rate.

Although one might expect an $O(\mathrm{Pe})$ correction to the osmotic pressure, general considerations show that at equilibrium $\langle\mathbf{B}\rangle$ must be proportional to the isotropic tensor, which contracts with $\langle\mathbf{E}\rangle$ to give zero. (The trace of $\mathbf{E}$ is zero from the incompressibility of the material.) Thus, a nonzero, nonisotropic $\langle\mathbf{B}\rangle$ must be proportional to $\langle\mathbf{E}\rangle$, and hence proportional to $\mathrm{Pe}$. Since the rate-of-strain contribution to the osmotic pressure is already proportional to (E) [cf. (17)], it is $O\left(\mathrm{Pe}^{2}\right)$. This $O\left(\mathrm{Pe}^{2}\right)$ rate-of-strain contribution can be determined from the $O(\mathrm{Pe})$ deformation to the structure, which we now do for a dilute suspension of Brownian hard spheres.

In a similar way, the $O(\mathrm{Pe})$ deformation to the structure results in a contribution to $\langle\boldsymbol{\nabla} \cdot \mathbf{A}\rangle$ that averages to the isotropic tensor times $\langle\mathbf{E}\rangle$, and this again gives zero. Presumably, the next term in the development of the deformation of the microstructure is $O\left(\mathrm{Pe}^{2}\right)$, and this would then give a nonzero, $O\left(\mathrm{Pe}^{2}\right)$, Brownian contribution to the osmotic pressure. Here we shall only calculate the $O(\mathrm{Pe})$ deformation to the microstructure, and thus only the $O\left(\mathrm{Pe}^{2}\right)$ rate-of-strain contribution to the osmotic pressure.

For a dilute suspension of Brownian hard spheres, Batchelor ${ }^{13}$ showed that it is sufficient to consider the interactions between only two particles alone in the fluid. The relevant microstructural quantity is the pairdistribution function $g(\mathbf{r})$, which satisfies the following Smoluchowski equation:

$$
\boldsymbol{\nabla} \cdot \mathbf{D} \cdot \nabla \mathbf{g}-\operatorname{Pe} \boldsymbol{\nabla} \cdot \mathbf{U} g=0,
$$

with

$$
\begin{aligned}
& g \sim 1, \quad \text { as } r \rightarrow \infty, \\
& \mathbf{n} \cdot(\mathbf{D} \cdot \nabla g-\mathrm{Pe} \cdot \mathrm{U} g)=0, \quad \text { at } r=2 .
\end{aligned}
$$

Here, $\mathbf{D}=k T \mathbf{R}_{F U}^{-1}$ is the relative diffusivity of two particles and $\mathbf{U}$ is the relative velocity due to the imposed shear flow, $\mathbf{U}=\mathbf{R}_{F U}^{-1} \cdot \mathbf{R}_{F E}:\langle\mathbf{E}\rangle$. [Care must be taken to ensure that the appropriate combinations of $\mathbf{R}_{F U}^{-1}$, etc. for two particles are taken to form the relative diffusivity and ve- 
locity in (20).] All lengths have been made dimensionless by the particle radius $a$, the velocity by $\dot{\gamma} a$, and the time by the diffusive time scale $a^{2} / D_{0}$, where $D_{0}=k T / 6 \pi \mu a$ is the diffusivity of an isolated particle. The Péclet number in (20) measures the relative importance of shear and Brownian forces.

The equilibrium solution of (20) is the Boltzmann distribution

$$
g_{0}=1 \text {, }
$$

to $O(\phi)$. The perturbation to the equilibrium structure will be linear in $\langle\mathbf{E}\rangle$, and therefore we define, writing $\langle\mathbf{E}\rangle \equiv \dot{\gamma}\langle\hat{\mathbf{E}}\rangle$,

$$
g=g_{0}\left[1-\operatorname{Pe} f(s) \frac{1}{2} \mathbf{d} \cdot\langle\hat{\mathbf{E}}\rangle \cdot \mathbf{d}\right],
$$

where, to leading order in $\mathrm{Pe}, f$ satisfies

$$
\frac{d}{d s}\left(s^{2} G \frac{d f}{d s}\right)-6 H f=-s^{2} W
$$

with

$$
G \frac{d f}{d s}=0, \quad \text { at } s=2 \text { and } f \rightarrow 0, \quad \text { as } \quad s \rightarrow \infty .
$$

Here $G$ and $H$ are the radial and tangential components of the relative diffusivity $\mathbf{D}$, and may be written in terms of the mobility functions relating velocity to force as

$$
G(s)=x_{11}^{a}(s)-x_{12}^{a}(s), \quad H(s)=y_{11}^{a}(s)-y_{12}^{a}(s),
$$

and $W$ is defined by

$$
\boldsymbol{\nabla} \cdot \mathbf{U}=W(s)(\mathbf{d} \cdot\langle\hat{\mathbf{E}}\rangle \cdot \mathbf{d}) .
$$

Batchelor ${ }^{13}$ wrote $W$ in terms of two functions $\mathscr{A}$ and $\mathscr{B}$ (Batchelor and Green ${ }^{14}$ ), which relate the rate of strain to the axisymmetric and nonaxisymmetric motions, respectively, for a pair of particles [note that $\mathscr{A}$ and $\mathscr{B} B$ are to be distinguished from $A$ and $B$ of (20)-(22)]:

$$
W(s)=3[\mathscr{B}(s)-\mathscr{A}(s)]-s \frac{d \mathscr{A}}{d s} .
$$

For equal-sized spheres, the functions $\mathscr{A}$ and $\mathscr{B}$ may be expressed in terms of nondimensionalized (see Kim and Karrila ${ }^{9}$ ) resistance and mobility functions as

$$
\mathscr{Z}(s)=\frac{4}{s}\left(x_{11}^{g}-x_{12}^{g}\right)=\frac{4}{3 s}\left(X_{11}^{G}-X_{12}^{G}\right)\left(x_{11}^{a}-x_{12}^{a}\right)
$$

and

$$
\begin{aligned}
\mathscr{B}(s)= & \frac{8}{s}\left(y_{11}^{g}-y_{12}^{g}\right) \\
= & \frac{8}{S}\left(\frac{1}{3}\left(y_{11}^{a}-y_{12}^{a}\right)\left(Y_{11}^{G}-Y_{12}^{G}\right)\right. \\
& \left.-\left(y_{11}^{b}-y_{12}^{b}\right)\left(Y_{11}^{H}+Y_{12}^{H}\right)\right),
\end{aligned}
$$

with $x_{\alpha \beta}^{g}$ and $y_{\alpha \beta}^{g}$ the functions relating the stresslet on particle $\alpha$ to the force on particle $\beta$ in the formulation of Kim and Mifflin, ${ }^{15} X_{\alpha \beta}^{G}$ and $Y_{\alpha \beta}^{G}$ the resistance functions relating force to rate of strain, and so forth. Equation (22)

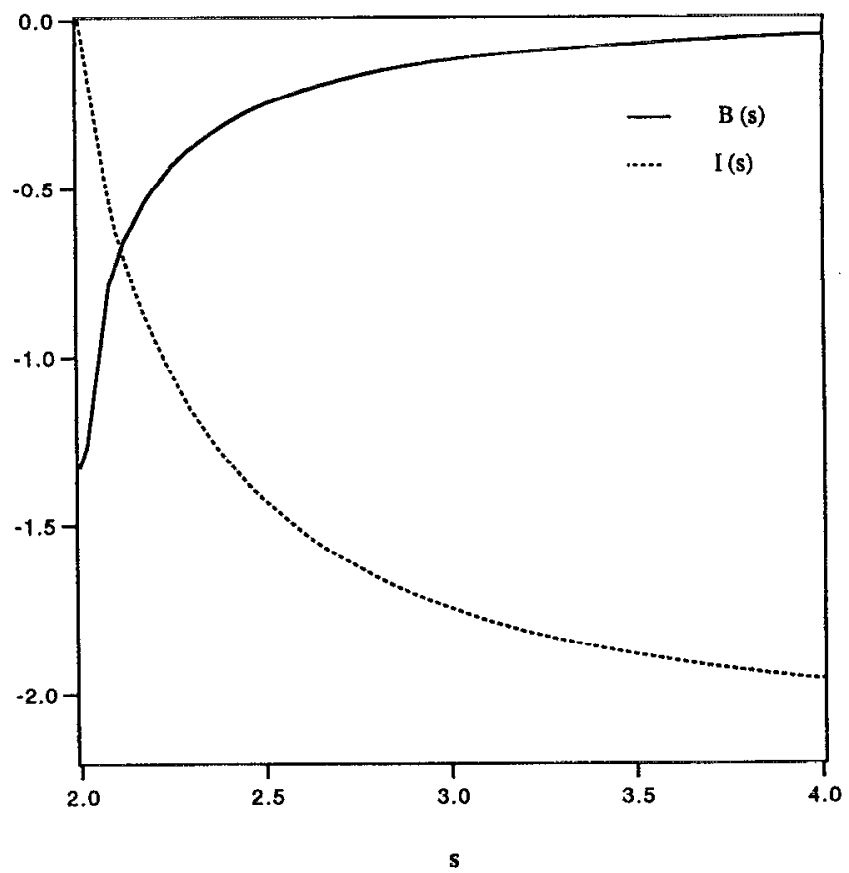

FIG. 3. The function $B(s)$, solid line, and $\int_{2}^{5} r^{2} f(r) B(r) d r$ from Eq. (23), dashed line labeled $I$, are plotted against dimensionless particle separation $s$. Here $B(s)$ has the value -1.33 at $s=2$ and the limiting value of $I$ as $s \rightarrow \infty$ is -2.1 .

was solved by Batchelor ${ }^{13}$ [a factor of $\frac{1}{2}$ in (21) is included so the form agrees with that work], and we have repeated the calculation here in order to have numerical values of $f$ for integration in (23) below. Note that $f$ decays as $s^{3}$ for large $s$.

In the hydrodynamic contribution to the osmotic pressure for two spheres, $\mathbf{B}$ can be expressed as

$$
\mathbf{B}=B(s)\left(\mathbf{d d}-\frac{1}{3} \mathrm{I}\right),
$$

where

$$
B(s)=\frac{8 \pi a^{3} \mu}{3}\left(\left(X_{11}^{P}-X_{12}^{P}\right) \frac{s \mathscr{A}(s)}{4}-\left(X_{11}^{Q}+X_{12}^{Q}\right)\right) .
$$

Since the perturbation to $g$ is proportional to dd: $\langle E\rangle$, the angular integration implied in the ensemble average of $\mathbf{B}$ will result in a term proportional to $\langle\mathbf{E}\rangle:\langle\mathbf{E}\rangle$. When $B(s)$ is normalized with $8 \pi a^{3} \mu / 3$ and the averaging is expressed as a probability integral over the pair-distribution function, we have

$$
\begin{aligned}
n\langle\mathbf{B}\rangle:\langle\mathbf{E}\rangle= & -\frac{1}{15 \pi} \frac{k T}{a^{3}} \mathrm{Pe}^{2} \phi^{2} \\
& \times \int_{2}^{\infty} B(s) f(s) s^{2} d s\langle\hat{\mathbf{E}}\rangle:\langle\hat{\mathbf{E}}\rangle .
\end{aligned}
$$

Note that the integral in (23) is absolutely convergent as $f \sim s^{-3}$ and $B(s) \sim s^{-3}$ for large $s$; also, note that the integrand is finite at contact, as the singularities in the $P$ and $Q$ functions cancel. Calculation of the integral gives

$$
n\langle\mathbf{B}\rangle:\langle\mathbf{E}\rangle=\frac{2.1}{15 \pi} \frac{k T}{a^{3}} \operatorname{Pe}^{2} \phi^{2}\langle\hat{\mathbf{E}}\rangle:\langle\hat{\mathbf{E}}\rangle .
$$


Alternatively, the result could be expressed as $0.84 \operatorname{Pe} \phi^{2} \mu \hat{\gamma}\langle\hat{\mathbf{E}}\rangle:\langle\hat{\mathbf{E}}\rangle$, corresponding to a hydrodynamic rather than thermal scaling of the stress. In Fig. 3, the nondimensional $B(s)$ and the integral on the right-hand side of (23) as a function of the upper limit of integration are plotted; the latter, labeled $I$, illustrates that the majority of the contribution is due to particles within two radii of the reference particle.

A brief consideration shows why the $\langle\mathbf{B}\rangle:\langle\mathbf{E}\rangle$ contribution is positive. Two particles approaching one another along the compressional axis in a straining flow must force fluid out of the intervening region, and the pressure moment for this interaction will be positive. For the case of two particles moving away from each other along an extensional axis, the moment is therefore negative, based on the reversibility of Stokes flow, and tangibly because fluid must be "sucked" into the region between the particles. The equilibrium structure is distorted by the weak straining, however, so that a second particle lies preferentially along the compressional axis relative to a reference particle, as will be seen from a study of the pair distribution (21). As a result, the average pressure from the interaction is positive.

\section{SUSPENSION PRESSURE IN A SHEARED LATTICE}

In this second example, we show the behavior of the pressure as a function of particle volume fraction for a sheared simple cubic lattice of spheres. The volume fractions illustrated are $\phi=0.10,0.30,0.41$, and 0.45 . For a suspension of force- and torque-free non-Brownian spheres, the suspension pressure is given by the rate-ofstrain contribution in (17) only:

$$
\Pi=\langle\mathbf{B}\rangle:\langle\mathbf{E}\rangle=\frac{1}{3}\left\langle\mathbf{P} \cdot \mathbf{R}_{F U}^{-1} \cdot \mathbf{R}_{F E}-\mathbf{Q}\right\rangle:\langle\mathbf{E}\rangle .
$$

Equation (24) is the general form for the rate-of-strain pressure in any suspension, not just a periodic lattice. For a random suspension, for example that given by a hardsphere microstructure, $\langle\mathbf{B}\rangle=\alpha(\phi) \mathbf{I}$, with $\mathbf{I}$ the isotropic tensor, and this contracts with $\langle\mathbf{E}\rangle$ to give zero. For an undeformed simple cubic lattice, $\langle\mathbf{B}\rangle$ is also proportional to $\mathbf{I}$, and there is no pressure. When the lattice is sheared along one of the lattice vectors, however, the instantaneous lattice is not simple cubic, but rather rhombohedral, and there is a nonzero pressure. As the lattice structure periodically repeats itself, the pressure must return to zero in onc period. For small volume fractions, this occurs by the pressure taking positive values in the first half of the cycle and then negative values for the second half, as shown in Fig. 4 for $\phi=0.10$ and $\phi=0.30$. The curves are antisymmetric about the midpoint of the cycle. At larger volume fractions, the values are seen to begin negative as the lattice is sheared away from registry; the curve is always antisymmetric about the midpoint of the cycle. This change of sign in the trace of the first moment as the particle fraction is increased may possibly be related to the negative secondorder coefficient in the series expansion in $\phi$ of the viscosity for a simple cubic lattice. ${ }^{16}$ We see the pressure increases with volume fraction as expected [the pressure is $O\left(\phi^{2}\right)$ at

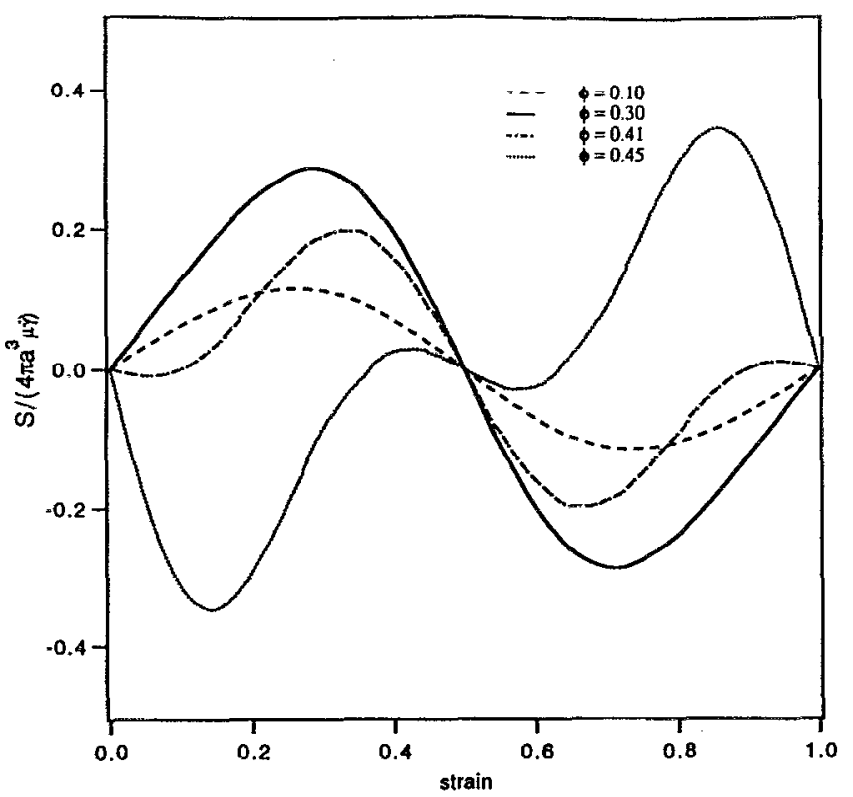

FIG. 4. Values of the trace of the first moment of the force distribution upon a sphere (of radius $a$ ) are plotted as a function of total strain for an initially simple cubic lattice undergoing simple shear flow at shear rate $j$ in a fluid of viscosity $\mu$; the motion is along a lattice vector. Volume fractions shown are $\phi=0.10,0.30,0.41$, and 0.45 . The lattice is at registry at zero strain and returns to registry first at a strain of unity. The trace is identically zero at registry and the midpoint of the cycle. The values are antisymmetric about the midpoint, and hence average to zero for a cycle; note the change of sign with incipient strain occurring near $\phi \doteq 0.41$.

low $\phi$; we have plotted the instantaneous value of the pressure moment for a chosen particle, so the dependence on $\phi$ is linear], although the change in sign at incipient strain at around $\phi=0.41$ leads to small values of the pressure for volume fractions near $\phi=0.41$.

The procedure used to calculate the pressure interactions for the lattice structure involves an accounting for near- and far-field interactions essentially equivalent to that used in the Stokesian dynamics method for evaluating. the grand resistance tensor. ${ }^{6}$ Expanding the force density as a series of moments about the particle centers in the integral formulation for the pressure, 9,10 a "far-field" estimate for the pressure at the particle of interest due to the moments on the other particles is calculated. We truncate the moment expansion at the level of the stresslet, which is calculated by Stokesian dynamics. When the interparticle distance is small, there is significant error in this approximation. For close pairs, the resistance functions $\mathbf{P}$ and $\mathbf{Q}$ reported here are used to calculate the trace for the particles, as prescribed by (3); in the flow of the lattice, there is no deviation of particle velocities from the bulk flow, so only $\mathbf{Q}: \mathbf{E}$ contributes. The contributions to the pressure at a reference sphere by all neighbors within a distance $s=4$ are calculated in this manner $(s=4$ being an arbitrary choice), and are added to the far-field estimate. For close pairs, this double counts that portion of the near-field interaction which is captured by the truncated moment expansion. The leading error in the moment expansion is due to neglect of the change to the pressure environment of a 
reference sphere by the induced quadrupole upon a neighboring sphere, which behaves as $s^{-8}$. Hence, we subtract from the sum the terms in the resistance formulation of $O\left(s^{-7}\right)$ and larger. It should be noted that the pressure environment experienced by a particle due to the stresslet on a second particle behaves as $s^{-3}$, with $s$ the separation distance; the interactions would be nonconvergent if simply summed pairwise. Thus, O'Brien's method ${ }^{17}$ for renormalization of nonconvergent hydrodynamic interactions was applied, with Ewald summation employed to speed convergence of the resulting expression (see Beenakker ${ }^{18}$ ).

\section{CONCLUDING REMARKS}

The functions presented here make it possible to calculate the isotropic stress associated with rigid particle interactions in low-Reynolds-number flow. As the examples in the preceding sections illustrate, the functions may be applied in either analytical or computational contexts. These examples were restricted to systems where the particle phase was under the influence of hydrodynamic and Brownian forces only. In general, suspensions with interparticle forces (electrostatic, for example) will have a stress contribution associated with these forces. Hence, (14) will be replaced by

$$
\langle\Sigma\rangle=-n k T \mathbf{I}+2 \mu\langle\mathbf{E}\rangle+n\left[\left\langle\mathbf{S}^{E}\right\rangle+\left\langle\mathbf{S}^{B}\right\rangle+\left\langle\mathbf{S}^{P}\right\rangle\right],
$$

with

$$
\left.\left\langle\mathbf{S}^{P}\right\rangle=-\left\langle\mathbf{R}_{S U} \cdot \mathbf{R}_{F U}^{-1}+\mathbf{x I}\right) \cdot \mathbf{F}^{P}\right\rangle,
$$

where $\mathrm{F}^{P}$ is the total (nonhydrodynamic) force exerted by the other particles upon the reference particle. The suspension pressure, mechanically defined to be negative onethird of the trace of the bulk stress, for a system with hydrodynamic, Brownian, and specific interparticle forces is

$$
\begin{aligned}
\Pi= & n k T+n k T\langle\nabla \cdot \mathbf{A}\rangle+n\left\langle\mathbf{x} \cdot \mathbf{F}^{P}\right\rangle+n\langle\mathbf{B}\rangle:\langle\mathbf{E}\rangle \\
& +n\left\langle\mathbf{A} \cdot \mathbf{F}^{P}\right\rangle,
\end{aligned}
$$

with (18) and (19) for $\mathbf{A}$ and $\mathbf{B}$. The $n\left\langle\mathbf{x} \cdot \mathbf{F}^{P}\right\rangle$ is the familiar $x F^{P}$ pressure, just as found in molecular systems, and the last two terms are the contributions from hydrodynamics.

Efforts to model two-phase fiow have made use of the concept of "particle-phase pressure." This quantity has not, to date, been placed on firm footing for viscously dominated suspensions; authors have given heuristic arguments for the scaling of the pressure with respect to system parameters ${ }^{5}$ or have stated that the particle interactions give rise to a positive pressure, ${ }^{3}$ without apparent justification. With the functions presented here, the pressure interactions of suspended particles in Stokes flow may be calculated. The roles of the strain rate and particle velocities are made clear, as is the fact that an induced pressure requires at least two particles. This work will, it is hoped, provide a means for rational investigation of the role of the particle-phase pressure in macroscopic models of twophase flow.

\section{ACKNOWLEDGMENTS}

This work was supported by the Natural Sciences and Engineering Research Council of Canada, the National Science Foundation Fellowship program, and the Office of Naval Research, under Grant No. N00014-90-J-1945.

'J. F. Brady, "Brownian motion, hydrodynamics and the osmotic pressure," J. Chem. Phys. 98, 3335 (1993)

${ }^{2}$ G. B. Wallis, One-Dimensional Two-Phase Flow (McGraw-Hill, New York, 1969).

${ }^{3} \mathrm{G}$. K. Batchelor, "A new theory of the instability of a uniform fluidized bed," J. Fluid Mech. 193, 75 (1988).

${ }^{4}$ D. L. Koch, "Kinetic theory for a monodisperse gas-solid suspension," Phys Fluids A. 2, 1711 (1990).

${ }^{5}$ P. R. Nott and J. F. Brady, "Pressure-driven suspension flow: Theory and simulation," submitted to J. Fluid Mech.

${ }^{6}$ J. F. Brady and G. Bossis, "Stokesian Dynamics," Annu. Rev. Fluid Mech. 20, 111 (1988).

${ }^{7}$ D. J. Jeffrey and Y. Onishi, "Calculation of the resistance and mobility functions for two unequal rigid spheres in low-Reynolds-number flow," J. Fluid Mech. 139, 261 (1984).

${ }^{8} \mathrm{D}$. J. Jeffrey, "The calculation of the low Reynolds number resistance functions for two unequal spheres," Phys. Fluids A 4, 16 (1992).

${ }^{9} \mathrm{~S}$. Kim and S. J. Karrila, Microhydrodynamics: Principles and Selected Applications (Butterworth, Boston, 1991).

${ }^{10} \mathrm{O}$. A. Ladyzhenskaya, The Mathematical Theory of Viscous Incompressible Flow (Gordon and Breach, New York, 1963).

${ }^{11} \mathrm{D}$. J. Jeffrey and R. M. Corless, "Forces and stresslets for the axisymmetric motion of nearly touching unequal spheres," Physicochem. Hydrodyn. 10, 461 (1988).

${ }^{12} \mathrm{D}$. J. Jeffrey, "Higher-order corrections to the axisymmetric interactions of nearly touching spheres," Phys. Fluids A 1, 1740 (1989).

${ }^{13} \mathrm{G}$. K. Batchelor, "The effect of Brownian motion on the bulk stress in a suspension particles," J. Fluid Mech. 83, 97 (1977).

${ }^{14} \mathrm{G}$. K. Batchelor and J. T. Green, "The hydrodynamic interactions of two small freely-moving spheres in a linear flow field," J. Fluid Mech. 56, 401 (1972).

${ }^{15} \mathrm{~S}$. Kim and R. T. Mifflin, "The resistance and mobility functions of two equal spheres in low-Reynolds-number flow," Phys. Fluids 28, 2033 (1985).

${ }^{16} \mathrm{~K}$. C. Nunan and J. B. Keller, "Effective viscosity of a periodic suspension," J. Fluid Mech. 142, 269 (1984).

${ }^{17} \mathrm{R}$. W. O'Brien, "A method for the calculation of the effective transport properties of interacting particles," J. Fluid Mech. 139, 261 (1979).

${ }^{18}$ C. W. J. Beenakker, "Ewald sum of the Rotne-Prager tensor," J. Chem. Phys. 85, 1581 (1986). 